\title{
Research on Architectures for Integrated Speech/Language Systems in Verbmobil
}

\author{
Günther Görz, Marcus Kesseler, Jörg Spilker, Hans Weber \\ University of Frlangen-Nürnberg \\ IMMD (Computer Science) VIII -- Artificial Intelligence \\ Am Weichselgarten 9 \\ D-91058 ERLANGEN \\ Email: \{goerz,kesseler, spilker, weber\}@informatik .uni-erlangen.de
}

\begin{abstract}
The German joint research project Verbmobil (VM) aims at the development of a speech to speech translation system. This paper reports on research done in our group which belongs to Verbrnobil's subproject on system architectures (TP15). Our specific research areas are the construction of parsers for spontaneous speech, investigations in the parallelization of parsing and to contribute to the development of a flexible com munication architecture with distributed control.
\end{abstract}

\section{Introduction}

The German joint research project Verbmobil $(\mathrm{VM})^{1}$ aims at the development of a speech to speech translation system. This paper reports on research done in our group which belongs to Verbmobil's subproject on system architectures (TP15). The task of this subproject is to provide basic research results on incremental and interactive system architectures for the VM research prototype and to demonstrate their feasibility in the prototypical INTARC system. Our specific research areas are the construction of parsers for spontaneous speech, investigations in the parallelization of parsing and to contribute to the development of a flexible communication architecture with distributed control. The paper is organized as follows: Section 2 reports on the design and implementation of an incremental interactive speech parser which integrates statistics

\footnotetext{
${ }^{1}$ This work was funded by the German Federal Ministry for Research and Technology (BMFT) in the framework of the Verbmobil Project under Grant BMFT 01 IV $101 \mathrm{H} / 9$. The responsibility for the contents of this study lies with the authors.
}

with a chart-parser employing a unification grammar (UG) formalism. Furthermore, results of experiments on the interaction between the parser and a speech recognizer using expectations, are reported. In section 3 we present experiences with a parallel version of the parser. Section 4 deals with distributed control in modular Natural Ianguage/Speech (NLSP) systems.

\section{Design and Implementation of Incremental Interactive Speech Parsers}

In a Left Right Incremental architecture (LRI), higher level modules can work in parallel with lower level modules. 'The obvious benefits of such an arrangement are twofold: 'The system does not have to wait for a speaker to stop talking and topdown constraints from higher level to lower level modules can be used easily. 'To achieve L RI behavior the singular modules must fulfill the following requirements:

Processing proceeds incrementally along the time axis ("left to right").

Pieces of output have to be transferred to the next module as soon as possible.

So far in INTARC-1.3 we have achieved an IRI style coupling of four different modules: Word recognition module, syntactic parser, semantic module and prosodic boundary module. Our word recognition module is a modified Viterbi decoder, where two changes in the algorithm design were made: We use only the forward search pass, and whenever a final HMM state is reached for an active word model, a corresponding word hypothesis is sent to the parser. Hence backward search becomes a part of the parsing algorithm. The LRI parsing algorithm is a modified active chart parser with an agenda driven control mechanism. 'Ihe chart vertices correspond to the frames of the signal representation. Edges correspond to word or phrase hypotheses, being partial in the case of ac- 
tive edges. A parsing cycle corresponds to a new time point related to the utterance. In every cycle a new vertex is created and new word hypotheses ending at that time point are read and inserted into the chart. In one cycle, a backward search is performed to the beginning of the utterance or to some designated time point in the past constituting a starting point for grammatical analysis. Search is guided by a weighted linear com bination of acoustic score, bigram score, prosody score, grammar derivation score and grammatical parsability. The search prodecure is a beam search implemented as an agenda access mechanism. 'The grammar is a probabilistic typed UG with separate rules for pauses and other spontanous speech phemomona.

\subsection{Basic: Objects}

In the following we use record notation to refer to subcomponents of an object. A chart vertex $V_{t}$ corresponds to frame number $t$. Vertices have four lists with pointers to edges ending in and starting in that vertex: inactive-out, inactivein, active-out and active-out. $\Lambda$ word hypothesis $W$ is a quadruple (from, to, key, score) with from and to being the start and end frames of $W$. $W . K e y$ is the name of the lexical entry of $W$ and $W . s c o r e$ is the acoustic score of $W$ for the frames spanned, given by a corresponding HMM acoustic word model. An edge $H_{3}$ consists of from, the start vertex and $t o$, a list of end vertices. Note that after a Viterbi forward pass identical word hypotheses do always come in sequence, differing only in ending time. Elactual is the last vertex added to F.to in an operation. 'Ihose "famnilies" of hypotheses are represented as one edge with a set of end vertices. Hiwords keeps the covered string of word hypotheses while SCORI is a record keeping score components. Besides that an edge consists of a grammar rule F. rule and E.next, a pointer to some olement of the right hand side of li.rule or NIL. $\Lambda$ s in standard active chart passing an edge is passive, if li.next $=$ nil, otherwise it is active. Ficat points to the left hand side of the grammar rule. SCORE is a record with entries for inside and outside probabilitics given to an edge by acoustic, bigram, prosody and grammar model:

Inside-X Model scores for the spanned portion of all edge.

Outside-X Optimistic estimates for the portion from vertex 0 to the beginning of an edge.

For every vertex we keep a best first store of scored edge pairs. We call that store Agenda $_{i}$ in cycle $i$.

\subsection{Basic Operations}

There are five basic operations to define the operations of the parsing algorithm. 'The two opcrations Combine and Seek Down are similar to the well known Earley algorithm operations Completer and Predictor. Furthermore, there are two operations to insert new word hypotheses, Insert and Inherit. All these operations can create now edges, so operations to calculate new scores from old ones are attached to them. In order to implement our beam search method appropriately but simply, we define an operation Agenda-Push, which selects pairs of active and passive edges to be pruned or to be processed in the future. The basic operations are given in Cla notation for simplicity.

\subsubsection{Combine}

For a pair of active and passive edges $(A, I)$, if $A$.neat $=$ l.cat and I.from $\in$ A.to, insert edge $L_{1}$ with E.rule $=$ A.rule, E.cat $:=$ A.cat, L.nexl $=$ shift (A.next), lifrom $=$ A. from, E. to $=$ A.to.

For $X=$ Bigram, Grammar and Prosody:

H.Outside $-X=$ A.Outside- $X+$ I.Inside- $X+$ $\operatorname{Trans}(X, A, 1)$

I.Inside- $X=A$.Inside- $X+$ I.Inside $X+$

$\operatorname{Trans}(X, A, L)$

For $X=\Lambda$ coustic:

E. Ontside- $X=A$. Outside $X[1$. from $] \oplus$ I.Inside $X$ $\operatorname{Trans}(X, A, I)$

L.Inside- $X=A$.Inside- $X[$ Ifrom] $\oplus$ I.Inside $-X$

$\operatorname{trans}(X, A, I)$

'The operator $(1)$ performs an addition of a number to every element of a set. $\operatorname{Trans}(X, A, L)$ is the specific transition penalty a model will give to two edges. In the case of acoustic scores, the penalty is always zero and can be neglected. In the bigram case it will be the transition from the last word covered by $A$ to the first word covered by $B$.

\subsubsection{Seek Down}

Whenever an active edge $A$ is inserted, insert an cdge $E$ for every rule $R$ such that $A$.next $=E$. cat , E. rule $=R$, D. from $=$ A actual, $E$. to $=\{$ A.actual $\}$

. For $X=\Lambda$ coustic, Prosody and Bigram:

L.Inside $X=0$

L.Outside- $X=-$ A.Outside- $X$

For $X=$ Grammar:

H.Inside- $X=$ grammar score of $R$

L. Outside- $X=A$.Outside $-X+\operatorname{Trans}(X, A, L)+$ F.Inside- $X$. 'This recursive operation of introducing new active edges is precompiled in our parser and extremely efficient. 


\subsubsection{Insert}

For a new word hypothesis $W=(a, i, k e y$, score $)$ such that no $W^{\prime}=(a, i-1, k e y$, score' $)$ exists, insert an edge $E$ with $E$.rule $=$ lex (key), E.cat $=$ lex(key), E.from $=V_{a}, E$.to $=\left\{V_{i}\right\}$ and for $X=$ Acoustic:

E.Inside- $X=$ E.Outside- $X=\{(i$, score $)\}$,

for $X=$ Prosody and Bigram:

E.Inside- $X=E$.Outside $-X=0$,

for $X=$ Grammar E.Inside- $X=$ E.Outside- $X=$ grammar score of lex(key).

\subsubsection{Inherit}

For a new word hypothesis $W=(a, i, k e y$, score $)$ such that a $W^{\prime}=(a, i-1, k e y$, score' $)$ exists:

For all $E$ in $V_{i-1}$.inactive-in or $V_{i-1}$.activein: If last(E.words) $=k e y$ then add $\left\{V_{i}\right\}$ to E.to, add (i,E.Inside-Acoustic[i-1] - score' + score) to E.Inside-Acoustic and add (i,E.OutsideAcoustic[i-1] - score' + score) to E.OutsideAcoustic.

If $E$ is active, perform a $S e e k$-Down on $E$ in $V_{i}$.

\subsubsection{Agenda Push}

Whenever an edge $E$ is inserted into the chart, if $E$ is active then for all passive $A$, such that A.from $\in$ E.to and combined-score $(E, A)>B$ BamValue, insert $(E, A$, combined-score $(E, A))$ into the actual agenda. If $E$ is passive then for all active $A$, such that E.from $\in A$.to and combined-score $(A, E)$ $>$ Beam-Value, insert (A,E,combined-score $(A, E))$ into the actual agenda. Combined-Score is a linear combination of the outside components of an edge $C$ which would be created by $A$ and $E$ in a Combine operation. Beam-Value is calculated as a fixed offset from the maximum Combined-Score on an agenda. Since we process best-first inside the beam, the maximum is known when the first triple is inserted into an agenda. Agenda-Pop will remove the best triple from an actual agenda and return it.

\subsection{A simple LRI lattice parser}

The follwing control loop implements a simple LRI lattice parser.

\section{1. $T=0$. Create $V_{T}$}

2. Insert initial active edge $E$ into $V_{T}$, with E. next $=S$

\section{Increment $T$. Create $V_{T}$}

4. For every $W$ with $W$.end $=' l$ ' $\operatorname{Insert}(W)$ or Inherit $(W)$

5. Until Agenda[T] is empty: (a) Combine (Agenda-Pop)

(b) When combination with initial edge is successful, send result to SEMANTICS

6. Communicate with PROSODY and go to 3

\subsection{The Grammar Model}

The UG used in our experiments consists of 700 lexical entries and 60 rules. We used a variant of inside-outside training to estimate a model of UG derivations. It is a rule bigram model similar to PCFG with special extensions for UG type operations. The probability of future unifications is made dependent from the result type of earlier unifications. The model is described in more detail in (Weber 1994a; Weber 1995); it is very similar to (Brew 1995).

\subsection{LRI Coupling with Prosody}

In INTARC we use three classes of boundaries, B0 (no boundary), B2 (phrase boundary), B3 (sentence boundary) and B9 (real break). The prosody module, developed at the University of Bonn, classifies time intervals according to these classes. A prosody hypothesis consists of a beginning and ending time and model probabilities for the boundary types which sum up to one. A prosodic transition penalty used in the Combine operation was taken to be the score of the best combination of bottom-up boundary hypothesis $B x$ and a trigram score (lword, $B x$, rword). Here lword is the last word of the edge to the left and rword is the first word spanned by the edge to the right. Prosody hypotheses are consumed by the parser in every cycle and represented as attributes of vertices which fall inside a prosodic time interval. In a couple of tests we already achieved a reduction of edges of about $10 \%$ without change in recognition rate using a very simple trigram with only five word categories.

\subsection{Experimental Results}

In a system like IN'TARC-1.3, the analysis tree is of much higher importance than the recovered string; for the goal of speech translation an adequate semantic representation for a string with word errors is more important than a good string with a wrong reading. The grammar scores have only indirect influence on the string; their main function is picking the right tree. We cannot measure something like a "tree recognition rate" or "rule accuracy", because there is no trcebank for our grammar. The word accuracy results cannot be compared to word accuracy as usually applied to an acoustic decoder in isolation. We counted only those words as recognized which could be 
built into a valid parse from the beginning of the utterance. Words to the right which could not be integrated into a parse, were counted as deletions -- although they might have been correct in standard word accuracy terms. 'This evaluation method is much harder than standard word accuracy, but it appears to be a good approximation to "rule accuracy". Using this strict method we achieved a word accuracy of $47 \%$, which is quite promising.

Results using top down prediction of possible word hypotheses by the parser -- work inspired by (Kita et. al. 1989) - have already been published in (Hauenstein and Weber 1994a; Haueristein and Weber 1994b), (Weber 1994a), and (Weber 1995). Recognition rates had been improved there for read speech. In spontaneous speech we could not achieve the same effects.

\subsection{Current Work}

Our current work, which led to INTARC-2.0, uses a new approach for the interaction of syntax and semantics and a revision of the interaction of the parser with a new decoder. For the last case we implemented a precompiler for word-based prediction which to our current experience is clearly superior to the previous word-class based prediction. For the implementation of the interaction of syntax and semantics we proceed as follows: A new turn-based UG has been written, for which a context-sensitive stochastic training is being performed. The resulting grammar is then stripped down to a pure type skeleton which is actually being used for syntactic parsing. Using full structure sharing in the syntactic chart, which contains only packed edges, we achieve a complexity of $O\left(n^{3}\right)$. In contrast to that, for semantic analysis a second, unpacked chart is used, whose edges are provided by an unpacker module which is the interface between the two analysis levels. The unpacker, which has exponential complexity, selects only the $n$ best scored packed edges, where $n$ is a parameter. Only if semantic analysis fails it requests further edges from the unpacker. In this way, the computational effort on the whole is kept as low as possible.

\section{$3 \quad$ Parallel Parsing}

One of our main research interests has been the exploration of performance gains in NLP through parallelization. To this end, we developed a parallel version of the INTARC parser. Although the results so far are yet not as encouraging as we expected, our efforts make for interesting lessons in software engincering. The parallel parser had to obey the following restrictions: Running on our local shared memory multiprocessor (SparcServer1000) with 6 processors, parallelization should be controlled by inserting Solaris 2.4 thread and process control primitives directly into the code. The only realistic choice we had was to translate our parser with Chestnut Inc.'s Lispto-C-Translator automatically into C. Since the Iisp functions library is available in $\mathrm{C}$ source, we could insert the necessary Solaris parallelisation and synchronization primitives into key positions of the involved functions.

\subsection{Parallelization Strategy and Preliminary Results}

For effective parallelization it is crucial to keep communication between processors to a minimum. Early experiments with a fully distributed chart showed that the effort required to keep the partial charts consistent was much larger that the potential gains of increased parallelism. 'The chart must be kept as a single data structure in a shared memory processor, where concurrent reads are possible and only concurrent writes have to be serialized with synchronisation primitives. An analysis of profiling data shows that even the heavily optimized UG formalism causes between $50 \%$-and $70 \%$ of the computational load in the scrial case. Therefore we provide an arbitrary number of unification workers running in parallel which are fed unification tasks from the top of an agenda sorted by scores. Duc to the high optimization level of the sequential parser, load-balancing is fairly poor. Namely, the very fast type check used to circumvent most unifications, causes large disparities in the granularity of agenda tasks. Furthermore, pathological examples have been found in which a single unification takes much longer than all other tasks combined.

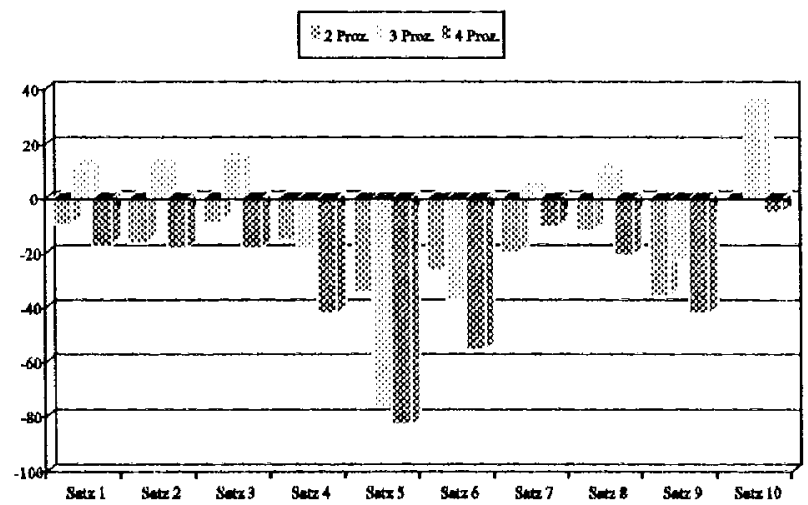

Figure 1: Percentual gains and losses over attained over 10 different sentences (Spilker 1995) 


\section{Distributed Control in Verbmobil}

The question of control in VM is tightly knit with the architecture of the VM system. As yet, the concept of architecture in VM has been used mostly to describe the overall modularization and the interfaces implied by the data flow between modules. This socalled domain architecture is incomplete in the sense that it does not specify any interaction strategies. Within our rescarch on interactive system architectures we developed a modular communication framework, $1 \mathrm{CE}^{2}$, in cooperation with the University of Hamburg. Now, ICE is the architectural frarnework of the VM research prototype.

\subsection{The INTARC Architecture}

The INTARC architecture as first presented by (Pyka 1992) is a distributed software system that allows for the interconnection of NLSP modules under the principles of incrementality and interactivity. Figure 2 shows the modularization of IN'TARC-1.3: There is a main broad channel connecting all modules in bottom-up direction, i.e., from signal to interpretation. Furthermore, there are smaller channels connecting several modules, which are used for the top-down interactive disambiguation data flow. Incrementality is required for all modules. ICE assumes that each module has a local memory that is not directly accessible to other modules. Modules communicate explicitly with one another via messages sent over bidirectional channels. 'This kind of communication architecture is hardly new and confronts us directly with a large number of unresolved issues in distributed problem solving, cf. (Durfee et al. 1989 ). In the last 20 years there have been numerous architecture proposals for distributed problem solving among computing entities that exchange information explicitly via message passing. None of these models include explicit strategies or paradigms to tackle the problem of distributed control.

\subsection{Structural Constraints of Verbmobil}

Modularity, being a fundamental assumption in VM (Wahlster 1992), does still lcave us with two problems: First, modules have to communicate with one another, and second, their local behaviors have to be somchow coordinated into a coherent global, possibly optimal, behavior. Unfortunately, the task of system integration has to obey some structural constraints which are mostly pragrnatic in nature:

\footnotetext{
${ }^{2}$ based on PVM (parallel virtual machine)
}

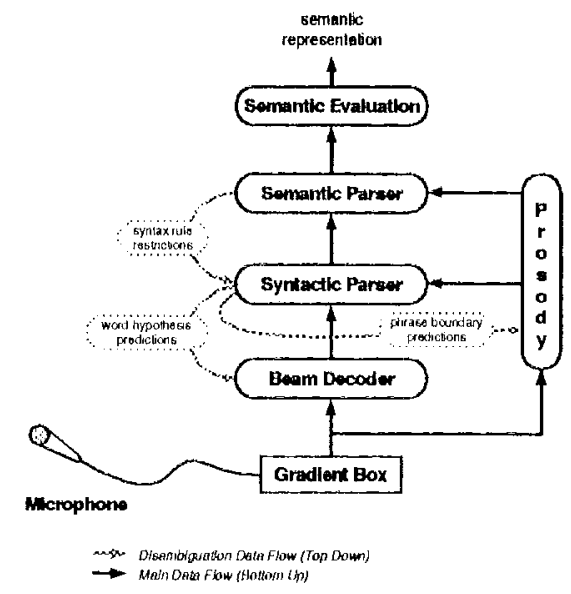

Figure 2: 'The interactive, incremental INTARC1.3 architecture

- Some of the modules are very complex software systems in themselves. Highly parameterizable and with control subtly spread over many interacting submodules, understanding and then integrating such systems into a common control strategy can be a very daunting task.

- Control issues are often very tightly knit with the domain the module is aimed at, i.e., it is very difficult to understand the control strategies used without sound knowledge of the underlying domain. The problem even gets worse if what is to be fine-tuned is the in teraction between several complex modules.

These two arguments are similar in nature, but differ in the architectural levels that they apply to. 'The former is implementation related, the latter algorithm and theory related.

\subsection{Layers of Control}

Modules have to communicate with one another and their local behaviors have to be coordinated into a coherent global, possibly optimal, behavior. In highly distributed systems we generally find the following levels of control:

System Control: 'The minimal set of operating system related actions that each participating module must be able to perform which will 
typically include means to start up, reset, monitor, trace and terminate individual modules or the systern as a whole.

Isolated Local Control: The control strategies used within the module disregarding any interactions beyond initial input of clata and final output of solutions. There is only one thread of control active at any time.

Interactive Local Control: Roughly, this can be seen as isolated local control extended with interaction capabilities. Incrementality is given by the possibility of control flowing back to a certain internal state after an output operation. Higher interactivity is made possible by entering a state more often from various points within the module and by adding a new waiting loop to check for any top-down requests. 'I he requirement for any. time betavior is a special case of that (Görz and Kesseler .1994).

In our experience the change to interactive control will tremendously increase the complexity of the resulting code. But we are still making the simplifying assumptions that the algorithm can be used incrementally -. but there are algorithms unsuitable for incremential processing (c.g. $A^{*}$ ). Incrementality can lead to the demand for a complete redesign of a module. Furthermore we assume that simply by exchanging data and doing simple extensions in the control flow everylthing will balance out nicely on the system scale which is enormously naive. Fven for the sequential architecture implied by the case of isolated local control, we have to solve a whole plethora of new problems that come along with interactivity;

- Mutual deadlock

- Mutual live-lock

- Race conditions (missing synchronization)

- Over-synchronization

Dialogue Control: In systems like VM there is a module that comes close to possessing the "inte. grated view" of a centralized blackboard control: the dialogue module. So it seems the right place to handle some of the global strategic control issues, like:

- 1)omain error handling

- Observe timeout constraints

- Resolve external ambiguitics/unknowns

'I'he fact that the dialogue module exercises a kind of global control does not invalidate what has been said about the unfeasability of central control, be. cause the control exereised by it is very coarse grained. 'I'o handle finer grained control issues in any module would take us back to memory and/or communication system contention.

\section{References}

Chris Brow. Stochastic HPSG. Procectings of the liuropean ACL, Conference 1995, Edinburgh, 1995

lidmund TI. Durfec, Victor R. Ixesser, and Danich 1). Corkill. Cooperative Distributed Problem Solving, pages 83 147. Volume 4 of Avron Barr et al. (Lid.). The Handbook of Artificial Intelligence Reading, Mass.: Addison-Wesley, 1989.

(iïnther Görz and Marcus Kesseler. Anytime Al gorithms for Spech l'arsing? Proceedings of (OLING-94, Kyoto, 1994

Andreas Haucnstein and llaus Weber. An investiga tion of tightly coupled time synchronous speech language interfaces using a unification grammar. In: I'. Mckevite (Ld.): P'roceedings of the Workshop on lntegration of Natural Language: and Speech Processing, NAAl 94, Suattle, 1994, 42-49

Andreas Hauenstein and Hans Weber. An Investigation of Tightly Coupled Time Synchronous Speech Language Interfaces. Procecdings of KONVFNS 94, Vienna: Springer, September 1994, 141 150

Marcus Kesseler. Distributed Control in Verbmobil. University of Frlangen-Nürnberg, IMMI) VIII, Verbmobil Report 24, 1994.

Kita, K., Kawabata, 'T. and Saito, IT. HMM contiuous spcech recognition using predictive $l, l$ parsing. IFEE' ICASSP' P'roccedings, 1989, 703-706.

Claudius Pyka. Management of hypolheses in an inlegrated specch-language archilecture. Proceedings of ECA 1-92, 1992, 558-560

Jörg Spilker. Parallelisierang eines inkrementellon, aktiven Chartparsers. Diploma thesis, University of Erlangen-Nürnberg, Frlangen, December 1995.

Wolfgang Wahlstor and Judith Jingelkamp, editors. Wissenschaftliche Ziele und Netzpläne für das VERBMOIII-Projekt. DFKI, Saarbrücken, 1992.

Hans Weber. Time Synchronous Chart Parsing of Spech Integrating Unification Grammars with Statistics. Speech and Language Fingineering, Pro ceedings of the Wighth T'wente Workshop on Lauguage T'cchnology, (1. l3oves, A. Nijholt, lid.), 'Iwente, 1994, 107-119

llans Weber. lik-inkrementelles probabilistisches Chartparsing von Worthypolhesenmengen mit Unifikationsgrammatiken: Eine enge Kopplung von Suche und Aralyse. Ph.J). 'I'hesis, University of Hamburg, 1995, Verbmobil Report 52. 